\title{
SN 2005 gj: evidence for LBV supernovae progenitors? ${ }^{\star}$
}

\author{
C. Trundle ${ }^{1}$, R. Kotak ${ }^{1}$, J. S. Vink ${ }^{2}$, and W. P. S. Meikle ${ }^{3}$ \\ 1 Astronomy Research Centre, Department of Physics \& Astronomy, School of Mathematics \& Physics, The Queen's University \\ of Belfast, Belfast BT7 1NN, Northern Ireland \\ e-mail: c.trundle@qub.ac.uk \\ 2 Armagh Observatory, College Hill, Armagh BT61 9DG, Northern Ireland \\ 3 Astrophysics group, Imperial College London, Blackett Laboratory, Prince Consort Rd, London SW7 2BZ, UK
}

Received 10 March 2008 / Accepted 15 April 2008

\begin{abstract}
There is mounting observational evidence in favour of Luminous Blue Variables (LBVs) being the direct progenitors of supernovae. Here we present possibly the most convincing evidence yet for such progenitors. We find multiple absorption component P-Cygni profiles of hydrogen and helium in the spectrum of SN 2005gj, which we interpret as being an imprint of the progenitor's mass-loss history. Such profiles have previously only been detected in Luminous Blue Variables. This striking resemblance of the profiles, along with wind velocities and periods consistent with those of LBVs leads us to connect SN 2005gj to an LBV progenitor.
\end{abstract}

Key words. stars: supernovae: general - stars: supernovae: individual: SN 2005gj - stars: supernovae: individual: SN 2002ic stars: circumstellar matter - stars: evolution - stars: winds, outflow

\section{Introduction}

In the canonical picture of the evolution of very massive $(M \gtrsim$ $40 M_{\odot}$ ) stars (e.g. Langer et al. 1994), the role of mass loss is widely accepted as being the key process that drives $\mathrm{O}$ and B-type stars into the luminous blue variable (LBV) phase, before entering the He-burning Wolf-Rayet (WR) stage. At the end of this phase, which lasts at least $10^{5}$ yrs (Meynet \& Maeder 2003; Eldridge \& Vink 2006), the object is expected to explode as a core-collapse supernova $(\mathrm{CcSN})$.

Of late, there has been a growing body of observational evidence that points to LBVs as the direct progenitors of a subset of CcSNe. Recently Kotak \& Vink (2006) suggested that LBVs themselves might explode; they proposed that the quasi-periodic modulations observed in the radio light curves of some SNe were a manifestation of variable mass loss during the $\mathrm{S}$ Doradus phase of LBVs, during which the star exhibits brightness variations of $\sim 1-2$ mag. This variability is commonplace amongst LBVs (Humphreys \& Davidson 1994) and is distinct from the giant outbursts such as those of P Cygni or $\eta$ Carina.

Interestingly, the progenitor of SN 2006jc was observed to have a giant eruption just two years prior to explosion (Nakano et al. 2006; Foley et al. 2007; Pastorello et al. 2007). Foley et al. (2007) argue that the progenitor star was an early type (WNE) Wolf-Rayet star. Given that WR stars have never previously been observed to undergo an LBV-like eruption, Pastorello et al. (2007) suggested a massive binary system consisting of an LBV and a WR star as an alternative progenitor system for SN 2006jc. In this scenario, the pre-SN explosion coincident with SN 2006jc is attributed to the LBV, while the SN resulted from the WR companion. A simpler explanation would be to

\footnotetext{
* Based on observations at the European Southern Observatory with UVES on the VLT in programme 276.D-5020A and 078.D-0720.
}

accept that a single, presumably massive star exploded during or at the very end of the LBV phase.

There are other indications that LBVs may explode. Gal-Yam et al. (2007) detected a very luminous source in preexplosion images of SN 2005gl, but it remains to be confirmed whether or not this is a single object. Other interesting hints come from the similarities in LBV nebula morphologies and the circumstellar medium of SN 1987A (Smith 2007). Finally, the luminous type IIn SN 2006gy may also have undergone an $\eta$ Car-type eruption prior to explosion (Smith et al. 2007).

A potential problem with a scenario in which LBVs explode is that current stellar evolution models do not predict core-collapse during or soon after the LBV phase; indeed, in most evolutionary models, the star has not even reached its core He-burning phase. In other words, in current models, the core is not evolved enough for core-collapse to occur. Pastorello et al. (2007) point out that known LBVs that have undergone outbursts still retain some hydrogen and helium while SN 2006jc showed no spectral features due to hydrogen before $\sim 65 \mathrm{~d}$. However, it is not inconceivable that the pre-explosion outburst removed most of the outer layers, and/or that residual amounts of $\mathrm{H}$ or He may not necessarily give rise to strong lines.

Here, we present the intriguing case of SN 2005gj for which we show spectroscopic evidence to support the suggestion of Kotak \& Vink (2006) that the variable mass-loss of S Dor variables reveals them as the endpoints of very massive stars.

\section{Data acquisition and reduction}

Optical spectra of SN 2005gj were obtained on 17 Dec. 2005 and 1 Oct. 2006, with the Ultra-Violet Echelle Spectrograph (UVES) on the Very Large Telescope. These epochs correspond to 86 and 374 days respectively, assuming an explosion date of 22 Sep. 2005 (Aldering et al. 2006). Dichroic \#2 was used to split the light into the blue and red arms of the spectrograph. The 

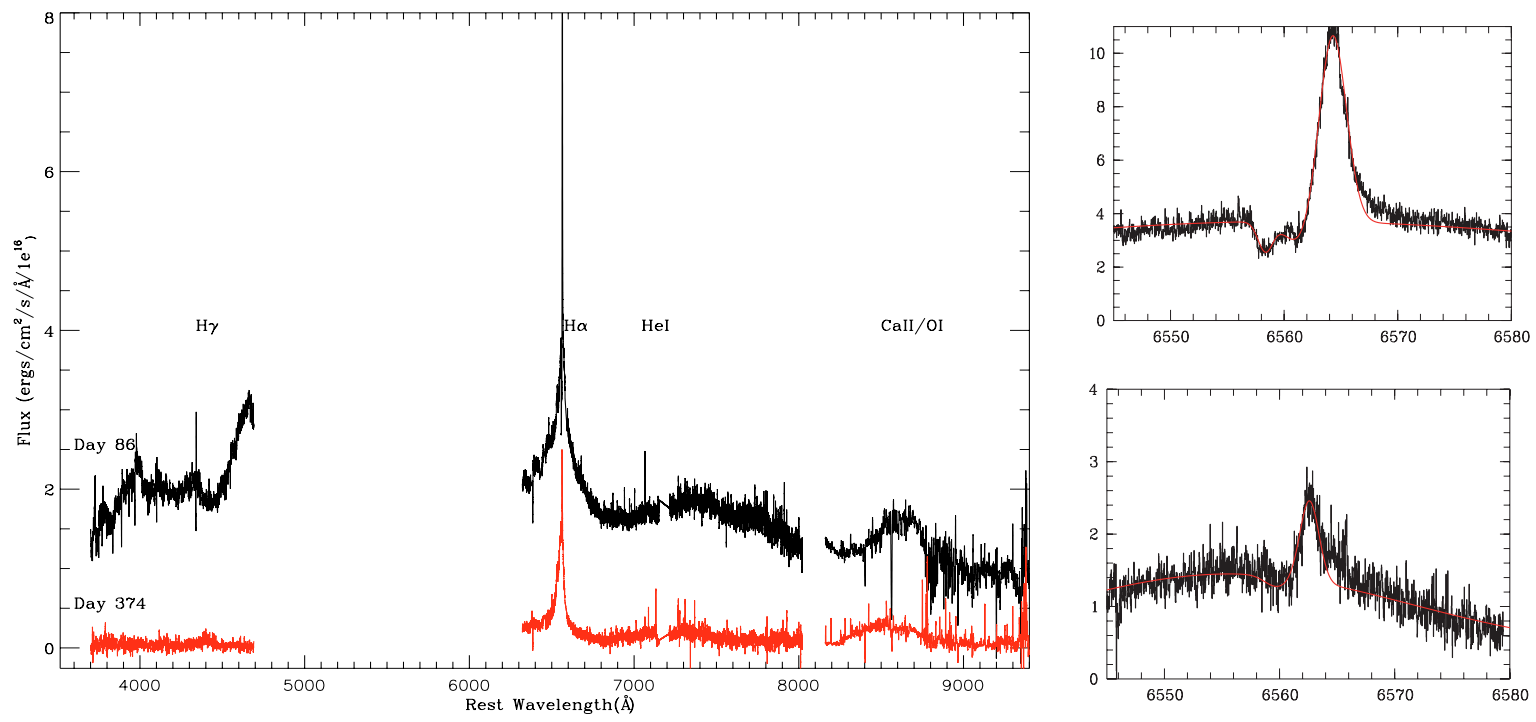

Fig. 1. Left: optical spectra of SN 2005gj at 86 and 374 days after explosion, after correction for the redshift of the host galaxy. Right: multiple component Gaussian profile fits to the H $\alpha$ profile for both epochs; day 86 in top panel and day 374 in bottom panel (Table 1). Units of the axes are the same as the left panel.

standard setup with central wavelengths at 4370 and $8600 \AA$ was employed, thus providing coverage in the blue of $3760-4985 \AA$, and in the red arms of $6705-8520 \AA$ and $8660-10255 \AA$. For both epochs the target was observed for $2300 \mathrm{~s}$ with a slit width of 1'.6 and a seeing of $\sim 1$ '. 05 providing resolutions of $\sim 6$ and $4.5 \mathrm{~km} \mathrm{~s}^{-1}$ in the blue and red arms, respectively.

The data were reduced with the UVES pipeline implemented in the ESO-MIDAS software. Wavelength calibration was carried out using Th-Ar arcs taken on the same night as the SN 2005gj exposures, while flux calibration and telluric subtraction of the data was done with respect to the standard LTT 1020. The flux calibrated spectra extracted from each chip and corrected to the rest frame are presented in Fig. 1. A redshift of $0.0616 \pm 0.0002$, as found by Aldering et al. (2006), was adopted as it was consistent with our data.

\section{Spectral analysis}

The optical spectra of SN 2005gj are dominated by $\mathrm{H} \alpha$ in emission. The profile consists of a strong narrow emission component superimposed on broad emission wings (Fig. 1). Such $\mathrm{H} \alpha$ profiles are the hallmarks of SN IIn spectra (" $n$ " standing for "narrow"), believed to arise from massive progenitors that have undergone substantial mass-loss prior to explosion (Schlegel 1990). The multi-component profiles are characterised by broad underlying emission due to $\mathrm{SN}$ ejecta, an intermediate emission resulting from shocked material at the interaction front between the ejecta and the CSM, while the narrow component presumably arises from unshocked CSM photoionised by the SN.

A striking feature of the P Cygni-like component of the $\mathrm{H} \alpha$ profile at $86 \mathrm{~d}$ is the presence of two clear absorption troughs (Fig. 1). This has previously not been observed in SN spectra. That this feature is associated with the SN is clear, given the evolution of the profile between the two epochs. Aldering et al. (2006) noted that the absorption component in the narrow P-Cygni profile was asymmetric and extended far out towards the blue edge. Due to the higher resolution of our spectra (cf. resolutions of a few hundred $\mathrm{km} \mathrm{s}^{-1}$, Aldering et al. 2006; Prieto et al. 2007), we were able to resolve this narrow component. Careful inspection of the data revealed clear double absorption
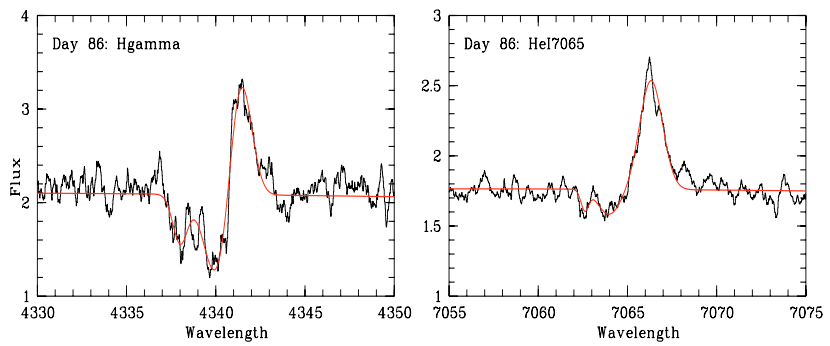

Fig. 2. Multiple Gaussian profile fits to the $86 \mathrm{~d}$ spectrum for $\mathrm{H} \gamma$ (left), and HeI $7065 \AA$ (right). The flux on the $y$-axis is given in units of $10^{-16} \mathrm{erg}^{-1} \mathrm{~cm}^{-2} \mathrm{~s}^{-1}$.

troughs in our day 86 spectrum associated with $\mathrm{H} \gamma$, He I $7065 \AA$ (Fig. 2), and also $\mathrm{H} \delta$, and He I $6078 \AA$ albeit with poorer signalto-noise ratios. By $\sim 400 \mathrm{~d}$, the $\mathrm{H} \alpha$ profile had changed significantly: the intensity dropped by $17 \times 10^{-16} \mathrm{erg}^{-1} \mathrm{~cm}^{-2} \mathrm{~s}^{-1}$, a single absorption trough remained, and a broad blue shoulder had developed, probably corresponding to the emergence of [O I]. To obtain quantitative information from these profiles we have decomposed them with multiple Gaussian profiles for the individual emission and absorption peaks. The fits are shown in Figs. 1 and 2 and their physical parameters are summarised in Table 1. The $\mathrm{H} \alpha$ emission profiles at both epochs were fit by 3 Gaussians representing the aforementioned broad, intermediate and narrow emission components, however this intermediate component was not detected in the $\mathrm{H} \gamma$ \& He I $7065 \AA$ profiles. In addition to the emission peaks, the $\mathrm{H} \alpha$ profile from day 86 was fit with double absorption components, whilst only a single absorption trough was required for the day 374 spectra.

Due to our incomplete wavelength coverage, particularly blueward of $\mathrm{H} \alpha$, the velocities determined from the Gaussian fits to the broad emission peak are subject to large uncertainties. From the Balmer lines we determine an ejecta velocity of $30000 \pm 5000 \mathrm{~km} \mathrm{~s}^{-1}$ at day 86 , which is consistent with that derived by Aldering et al. from their day 11 spectra. At day 374 the velocity decreased to $15500 \pm 5000 \mathrm{~km} \mathrm{~s}^{-1}$. The intermediate $\mathrm{H} \alpha$ emission component was fit by a Gaussian with $F W H M 2560 \pm 30 \mathrm{~km} \mathrm{~s}^{-1}$ on day 86 and $1680 \pm 30 \mathrm{~km} \mathrm{~s}^{-1}$ on 
Table 1. Parameters of the Gaussian fits to the narrow components of $\mathrm{H}_{\gamma, \alpha}$ \& He I $7065 \AA$ lines of SN 2005gj at 86 and 374 days after explosion. "Narrow E" - the narrow emission component, "NAC (a)" and "NAC (b)" - the blue and red absorption components respectively. Each profile has been fit by multiple Gaussian profiles, representing the broad, intermediate and narrow components of the profile.

\begin{tabular}{|c|c|c|c|}
\hline Fit properties & Narrow E & NAC (a) & $\overline{\mathrm{NAC}(\mathrm{b})}$ \\
\hline & \multicolumn{3}{|c|}{ Day 86: $\mathrm{H}_{\alpha}$} \\
\hline$\lambda(\AA)$ & $6564.29 \pm 0.02$ & $6558.35 \pm 0.06$ & $6561.25 \pm 0.06$ \\
\hline$F W H M\left(\mathrm{~km} \mathrm{~s}^{-1}\right)$ & $128 \pm 2$ & $70 \pm 6$ & $133 \pm 21$ \\
\hline$L\left(10^{40} \mathrm{erg} \mathrm{s}^{-1}\right)$ & $1.61 \pm 0.02$ & $0.14 \pm 0.01$ & $0.20 \pm 0.02$ \\
\hline \multirow[t]{2}{*}{$v_{\text {edge }}\left(\mathrm{km} \mathrm{s}^{-1}\right)$} & & $295 \pm 57$ & $120 \pm 75$ \\
\hline & \multicolumn{3}{|c|}{ Day 86: $\mathrm{H}_{\gamma}$} \\
\hline$\lambda(\AA)$ & $4341.39 \pm 0.02$ & $4338.01 \pm 0.10$ & $4340.04 \pm 0.10$ \\
\hline$F W H M\left(\mathrm{~km} \mathrm{~s}^{-1}\right)$ & $97 \pm 3$ & $68 \pm 5$ & $115 \pm 4$ \\
\hline$L\left(10^{40} \mathrm{erg} \mathrm{s}^{-1}\right)$ & $0.15 \pm 0.04$ & $0.04 \pm 0.01$ & $0.12 \pm 0.05$ \\
\hline \multirow[t]{2}{*}{$v_{\text {edge }}\left(\mathrm{km} \mathrm{s}^{-1}\right)$} & & $286 \pm 57$ & $115 \pm 64$ \\
\hline & \multicolumn{3}{|c|}{ Day 86: HeI7065 } \\
\hline$\lambda(\AA)$ & $7066.33 \pm 0.03$ & $7062.61 \pm 0.09$ & $7063.97 \pm 0.10$ \\
\hline$F W H M\left(\mathrm{~km} \mathrm{~s}^{-1}\right)$ & $64 \pm 3$ & $22 \pm 9$ & $62 \pm 15$ \\
\hline$L\left(10^{40} \mathrm{erg} \mathrm{s}^{-1}\right)$ & $0.10 \pm 0.02$ & $0.006 \pm 0.001$ & $0.022 \pm 0.004$ \\
\hline \multirow[t]{2}{*}{$v_{\text {edge }}\left(\mathrm{km} \mathrm{s}^{-1}\right)$} & & $160 \pm 59$ & $95 \pm 57$ \\
\hline & \multicolumn{3}{|c|}{ Day 374: $\mathrm{H}_{\alpha}$} \\
\hline$\lambda(\AA)$ & \multicolumn{3}{|c|}{$6562.60 \pm 0.046559 .70 \pm 0.12$} \\
\hline$F W H M\left(\mathrm{~km} \mathrm{~s}^{-1}\right)$ & \multirow{2}{*}{\multicolumn{3}{|c|}{$\begin{array}{c}110 \pm 23 \\
0.028 \pm 0.005\end{array}$}} \\
\hline$L\left(10^{40} \mathrm{erg} \mathrm{s}^{-1}\right)$ & & & \\
\hline$v_{\text {edge }}\left(\mathrm{km} \mathrm{s}^{-1}\right)$ & \multicolumn{3}{|c|}{$190 \pm 64$} \\
\hline
\end{tabular}

day 374. Whilst this measurement is a good indication of the shock velocity for the symmetric profile presented at day 86 , this is not the case for the asymmetric profile on day 374. In this later spectra the narrow $\mathrm{H} \alpha$ feature is redshifted with respect to the intermediate component; this has been seen in a number of type IIn spectra such as SN 1997ab, 1997eg \& the hybrid type Ia/IIn SN 2002ic (Kotak et al. 2004). This is generally attributed to self-absorption in the intermediate component which preferentially attenuates the red wing of the profile (Salamanca et al. 1998). A better measurement of the shock velocity is therefore the velocity of the blue edge of the profile at zero intensity, which is $2850 \pm 200 \mathrm{~km} \mathrm{~s}^{-1}$. The luminosities inferred from the narrow line intensities at day 86 agree well with those derived by Aldering et al., with a significant decline in the luminosities derived from the narrow component from $1.6 \times 10^{40} \mathrm{erg}^{-1}$ to $1.8 \times$ $10^{39} \mathrm{erg}^{-1}$ by day 374 .

Other, less dramatic features in the spectra include a broad feature at $\sim 8500 \AA$, probably a blend of the Ca II infrared triplet with O I 8446. There is a weak, broad feature centered on $7300 \AA$ which, depending on the SN type, can be identified as either [Ca II] or [Fe II], with the latter being present typically in the late-time spectra of SNe Ia. The [Ca II] lines however, are typically observed in the spectra of type IIn and Ib/c SNe at $\gtrsim 100 \mathrm{~d}$.

\section{SN 2005gj: properties of the unshocked CSM}

These narrow P-Cygni features, which are commonly found amongst type IIn, are thought to represent the outflow of unshocked CSM surrounding the SN event. As such, it provides insight into the environment prior to the SN explosion and on the wind properties of the SN progenitor itself. Stellar wind terminal velocities, $v_{\infty}$, are usually measured from the blue edge of strong resonance lines $\left(v_{\text {edge }}\right)$. Unfortunately, there are no such resonance lines in the optical, and so we will use the Balmer and $\mathrm{He} \mathrm{I}$ lines to estimate this velocity from their absorption components. As these lines are not saturated, $v_{\text {edge }}$ is likely to underestimate the terminal velocity (Prinja et al. 1990). From the $\mathrm{H} \alpha$ narrow absorption feature, we find a $v_{\text {edge }}$ of $295 \mathrm{~km} \mathrm{~s}^{-1}$ at $86 \mathrm{~d}$ with an additional component at $120 \mathrm{~km} \mathrm{~s}^{-1}$ whereas the component at $374 \mathrm{~d}$ has an intermediate velocity of $190 \mathrm{~km} \mathrm{~s}^{-1}$. The $\mathrm{H} \gamma$ feature at day 86 gives velocities consistent with those found from $\mathrm{H} \alpha$, however the bluer component of the He I profile appears at a lower velocity with a maximum $v_{\text {edge }}$ of $160 \mathrm{~km} \mathrm{~s}^{-1}$.

We can attempt to derive an estimate of the mass-loss rate from the $\mathrm{H} \alpha$ feature. Following Salamanca et al. (1998), $L_{\mathrm{H}_{\alpha}}^{\mathrm{Int}}=$ $\epsilon_{\mathrm{H}_{\alpha}} \dot{M} v_{\mathrm{S}}^{3} / 4 v_{\mathrm{w}}$ where, $\epsilon_{\mathrm{H}_{\alpha}}$ is an efficiency factor that peaks at $\sim 0.1$. $L_{\mathrm{H}_{\alpha}}^{\mathrm{Int}}$ is the luminosity of the intermediate component, $v_{\mathrm{w}}$ is the wind velocity and $v_{\mathrm{s}}$ is the shocked CSM velocity. At day 86, this implies $\dot{M}=6.4$ and $2.6 \times 10^{-2} M_{\odot} \mathrm{yr}^{-1}$ from the high $\left(295 \mathrm{~km} \mathrm{~s}^{-1}\right)$ and low $\left(120 \mathrm{~km} \mathrm{~s}^{-1}\right)$ velocity components. At day 374 , the derived $\dot{M}$ is $1.7 \times 10^{-2} M_{\odot} \mathrm{yr}^{-1}$ using $v_{\mathrm{w}}=$ $190 \mathrm{~km} \mathrm{~s}^{-1}$ and $v_{\mathrm{s}}=2850 \mathrm{~km} \mathrm{~s}^{-1}$. The shock velocity is a little less certain in this case (see Sect. 3). These are very high mass-loss rates but are typical of those derived from the $\mathrm{H} \alpha$ luminosity in supernovae, which show strong interaction between the ejecta and CSM (e.g. SNe 1997ab, 2002ic Salamanca et al. 1998; Kotak et al. 2004). However, Kotak et al. found that massloss rates derived from dust measurements in the infrared are a magnitude lower. A similar observation can be made by comparing the mass-loss rates of SN 2005gj derived here and those of Prieto et al. from X-ray luminosities. Several caveats must be borne in mind when estimating mass loss rates via $\mathrm{H} \alpha$ profiles. Two of the most serious shortfalls involve the unknown ionisation conditions and uncertainties in $\epsilon_{\mathrm{H}_{\alpha}}$. Realistic constraints can only be derived from detailed modelling which is beyond the scope of this work.

\section{Discussion}

SN 2005gj has been compared to the prototype of the hybrid type Ia/IIn objects, SN 2002ic (Aldering et al. 2006). SN 2002ic was initially classified as a type Ia, due to the presence of S II lines and a blue shifted absorption feature of Si II $6335 \AA$, but was quickly reclassified as a hybrid type Ia/IIn due to the presence of multi-component Balmer lines signifying interaction with the surrounding circumstellar medium (Hamuy et al. 2003). This was an exciting discovery as the presence of circumstellar hydrogen in the spectra of a type Ia supernovae would shed light on the nature of type Ia progenitors. One scenario put forward by Hamuy et al. is that the dense CSM could represent mass-loss from an asymptotic-giant-branch (AGB) star. Unfortunately, the true classification and interpretation of SN 2002ic remains under debate. In particular, Benetti et al. (2006) argue that it can equally well be compared to a core-collapse $\mathrm{SN}$, such as the type Ic SN 2004aw. This comparison is most convincing at late epochs; in fact the resemblance of SN 2002ic to type IIn SNe had been noted earlier (e.g. Hamuy et al. 2003).

In spite of the $\mathrm{H} \alpha$ feature indicating CSM interaction, the early time optical spectra of SN 2002ic were undeniably Ia-like (see Fig. 3 Hamuy et al. 2003). Aldering et al. (2006) and Prieto et al. (2007) argue that SN 2005gj could be compared to the overluminous type Ia, SN 1991 T "diluted" by the presence of significant CSM. However, the typical Ia spectral features due to S II and Si II which formed the crux of the Ia-evidence for SN 2002ic are barely discernible in the spectra of SN 2005gj. They attribute this to stronger interaction of the SN 2005gj with the CSM than for SN 2002ic, arguing that this claim is supported by its broader light curve and higher $\mathrm{H} \alpha$ luminosities. 


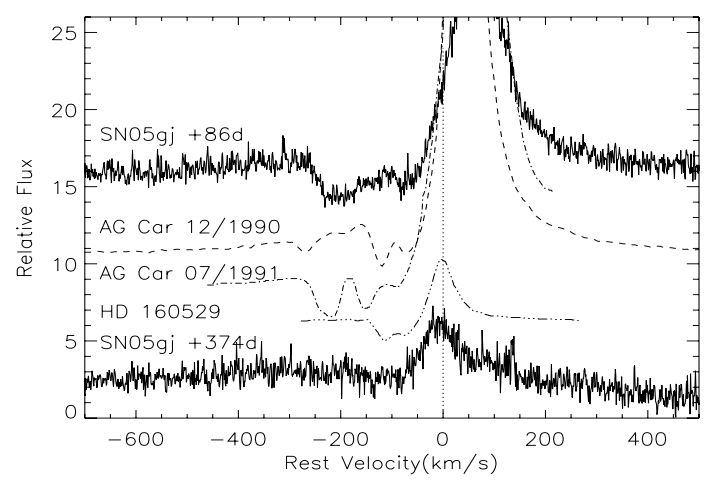

Fig. 3. Comparison of the $\mathrm{H} \alpha$ profile of SN 2005gj in velocity space with that of the luminous blue variables, AG Car \& HD 160529. Data taken from Stahl et al. (2001, 2003).

On the basis of the data presented here, we cannot verify or exclude the type Ia identification of SN 2005gj. However, we present an alternative scenario that is consistent with the observations. We focus our attention on the double absorption troughs seen in the day 86 spectrum. Only in one other SN spectrum, SN 1998S, have double absorption troughs been dectected (Bowen et al. 2000; Fassia et al. 2001). However the shape of the lines differs significantly. In SN 1998S a narrow P-Cygni profile of $\sim 50 \mathrm{~km} \mathrm{~s}^{-1}$ is superimposed on a broad, shallow absorption profile (Bowen et al. 2000, see Fig. 8). Additionally, this absorption decreases in relative intensity from lower to higher Balmer lines, suggestive of an optical depth dependence that is inconsistent with the SN 2005gj spectra. Chugai et al. (2002) invoke several mechanisms that could accelerate the circumstellar gas around SN 1998S. These models have been tailored to match the early-time spectra of SN 1998S and it is difficult to see how these might be applied to the significantly later epochs of our data. This type of test may provide some insight but is beyond the scope of this Letter.

The profiles in SN 2005gj bear a striking similarity to those in only one other stellar object: Luminous Blue Variables. Multiple-absorption component $\mathrm{H} \alpha$ profiles have been detected in many LBVs, viz AG Carinae, R127, R81, HD 160529, R66, \& P-Cygni (Wolf et al. 1981; Stahl et al. 1983a,b, 2001, 2003; Leitherer et al. 1994). Figure 3 shows several examples of the $\mathrm{H} \alpha$ profiles of LBVs gleaned from the literature. In LBVs these multiple-absorption component profiles signify their history of episodic mass-loss events. Vink \& de Koter (2002) interpreted the variable mass-loss and wind velocity behaviour of LBVs as being due to a change in the ionization of the dominant wind driving ion $\mathrm{Fe}$ (wind bistability). This mechanism was also invoked to explain the variations in the radio lightcurves of transitional SNe (Kotak \& Vink 2006). However, regardless of the mechanism, the resemblance of the double-troughed LBV $\mathrm{H} \alpha$ profiles to that of SN $2005 \mathrm{gj}$ remains.

An interesting hydrodynamical simulation by van Marle et al. (2007) shows the evolution of CSM around a $60 M_{\odot}$ star as it evolves from main-sequence to core-collapse via an LBV and WR phase. This simulation shows the formation of multiple absorption components from CSM shells formed by a fast WR wind running into an LBV shell. However these multiple shells occur in the very early stage of the WR-lifetime and hence if such a scenario was implied for SN 2005gj, its WR-phase would have been short-lived.

While the morphological resemblance of the line profiles observed in SN 2005gj and in LBVs is impressive enough, an even more compelling similarity is that of LBV wind velocities to those derived from the absorption components of SN 2005gj. LBV velocities lie in the $\sim 50-300 \mathrm{~km} \mathrm{~s}^{-1}$ range, in strikingly good agreement with those derived from SN 2005gj (Table 1), which are larger than those observed in AGB winds $\left(\sim 10 \mathrm{~km} \mathrm{~s}^{-1}\right)$ and red-supergiant winds and much lower than typical WR-star winds. Typical timescales of $\mathrm{S}$ Doradus mass-loss events in LBVs are on the scale of years up to 100 yrs (van Genderen 2001). From a simple calculation we can determine the timescales of the profile variations detected in SN 2005gj. Using an intermediate ejecta velocity of $22500 \mathrm{~km} \mathrm{~s}^{-1}$, a time variation of 288 days and an edge velocity of $300 \mathrm{~km} \mathrm{~s}^{-1}$ we get $P_{\mathrm{w}}=R_{\text {ejecta }} / v_{\text {edge }} \sim 60 \mathrm{yrs}$. Thus the timescales associated with mass-loss events in LBV and SN progenitors are consistent. Note that the $60 \mathrm{yr}$ timescale is only an upper limit as we have not obtained a well-sampled temporal dataset of high-resolution spectra. Hence the variations are likely to be on shorter timescales still consistent with S Doradus variations. Even the change into a single absorption component (374 d) mimics the behaviour of LBVs such as AG Car which show similar variations on timescales of years (Fig. 3).

For SN 2005gj the line profiles, wind velocities and wind periodicity are remarkably reminiscent of LBVs and provide significant support for a direct link between supernovae and luminous blue variables. This may have a huge impact on our understanding of massive star evolution since current theory predicts that LBVs, which are in a short-term, intermediary phase of evolution, should not undergo core-collapse.

Acknowledgements. The authors acknowledge fruitful discussions with L. Dessart and N. Langer.

\section{References}

Aldering, G., Antilogus, P., Bailey, S., et al. 2006, ApJ, 650, 510 Benetti, S., Cappellaro, E., Turatto, M., et al. 2006, ApJ, 2, L129 Bowen, D. V., Roth, K. C., Meyer, D. M., \& Blades, J. C. 2000, ApJ, 536, L225 Chugai, N. N., Blinnikov, S. I., Fassia, A., et al. 2002, MNRAS, 330, 473 Eldridge, J. J., \& Vink, J. S. 2006, A\&A, 452, 295

Fassia, A., Meikle, W. P. S., Chugai, N. N., et al. 2001, MNRAS, 325, 907 Foley, R. J., Smith, N., Ganeshalingam, M., et al. 2007, ApJ, 657, L105 Gal-Yam, A., Leonard, D. C., Fox, D. B., et al. 2007, ApJ, 656, 372 Hamuy, M., Phillips, M. M., Suntzeff, N. B., et al. 2003, Nature, 424, 651 Humphreys, R. M., \& Davidson, K. 1994, PASP, 106, 1025

Kotak, R., \& Vink, J. S. 2006, A\&A, 460, L5

Kotak, R., Meikle, W. P. S., Adamson, A., \& Leggett, S. K. 2004, MNRAS, 354, L13

Langer, N., Hamann, W.-R., Lennon, M., et al. 1994, A\&A, 290, 819

Leitherer, C., Allen, R., Altner, B., et al. 1994, ApJ, 428, L292

Meynet, G., \& Maeder, A. 2003, A\&A, 404, 975

Nakano, S., Itagaki, K., Puckett, T., \& Gorelli, R. 2006, Cent. Bur. Electron. Tel., 666,1

Pastorello, A., Smartt, S. J., Mattila, S., et al. 2007, Nature, 447, 829

Prieto, J. L., Garnavich, P. M., Phillips, M. M., et al. 2007, AJ, submitted

Prinja, R. K., Barlow, M. J., \& Howarth, O. D. 1990, ApJ, 361, 607

Salamanca, I., Cid-Fernandes, R., Tenorio-Tagle, G., et al. 1998, MNRAS, 300, L17

Schlegel, E. M. 1990, MNRAS, 244, 269

Smith, N. 2007, AJ, 133, 1034

Smith, N., Li, W., Foley, R. J., et al. 2007, ApJ, 666, 1116

Stahl, O., Wolf, B., Zickgraf, F.-J., et al. 1983a, A\&A, 120, 287

Stahl, O., Wolf, B., Klare, G., et al. 1983b, A\&A, 127, 49

Stahl, O., Jankovics, I., Kovács, J., et al. 2001, A\&A, 375, 54

Stahl, O., Gäng, T., Sterken, C., et al. 2003, A\&A, 400, 279

Wolf, B., Stahl, O., de Groot, M. J. H., \& Sterken, C. 1981, A\&A, 99, 351

van Genderen, A. M. 2001, A\&A, 366, 508

van Marle, A. J., Langer, N., \& García-Segura, G. 2007, A\&A, 469, 948

Vink, J. S., \& de Koter, J. S. 2002, A\&A, 393, 543 